\title{
Corela
}

Cognition, représentation, langage

HS-10 | 2012

Paramétrer le sens ? Études de cas

\section{Coordination et sens relationnel : formes schématiques, paramètres et instructions de cible}

\section{Martine Sekali}

\section{(2) OpenEdition}

\section{Journals}

Édition électronique

URL : http://journals.openedition.org/corela/2381

DOI : 10.4000/corela.2381

ISSN : $1638-573 \mathrm{X}$

\section{Éditeur}

Cercle linguistique du Centre et de I'Ouest - CerLICO

\section{Référence électronique}

Martine Sekali, «Coordination et sens relationnel : formes schématiques, paramètres et instructions de cible », Corela [En ligne], HS-10 | 2012, mis en ligne le 30 janvier 2012, consulté le 02 mai 2019. URL : http://journals.openedition.org/corela/2381 ; DOI : 10.4000/corela.2381

Ce document a été généré automatiquement le 2 mai 2019.

\section{(c) (i) (2)(2)}

Corela - cognition, représentation, langage est mis à disposition selon les termes de la licence Creative Commons Attribution - Pas d'Utilisation Commerciale - Partage dans les Mêmes Conditions 4.0 International. 


\title{
Coordination et sens relationnel : formes schématiques, paramètres et instructions de cible
}

\author{
Martine Sekali
}

\section{Préambule : les invariants sont-ils variables ?}

1 Les coordonnants sont des morphèmes linguistiques glissants : qu'on les approche sous l'angle de la syntaxe, et ils sont réputés former une catégorie de structures nonhiérarchisées. Les mêmes morphèmes considérés au niveau conceptuel (sémantique), peuvent, selon les contextes dans lesquels ils s'insèrent, définir une hiérarchie des domaines notionnels, dans des structures sémantiquement asymétriques. Le coordonnant anglais AND, par exemple, construit, dans l'énoncé (1), une structure nommée 'coordonnée' en syntaxe, mais une relation asymétrique (et donc subordonnante) en sémantique :

(1) You put me back in jail and I'm finished. I'm a dead man. (Auster, Brooklyn, 207)

2 AND marque ici une relation de dépendance conceptuelle de type conditionnel, paraphrasable en 'If you put me back I jail, I'm finished'. Ce phénomène, bien connu des linguistes (cf. en particulier Delechelle 1994, Culicover \& Jackendoff 1997, Trévise \& Constant 2007), est également représenté dans l'exemple (2) :

(2) This is my restaurant, and nobody tells me what to do! (Auster, Brooklyn, 122)

AND y explicite une relation déjà interprétable, du fait de la nature des notions complexes internes aux propositions, comme un lien de cause à conséquence, donc sémantiquement asymétrique, puisque marquant une dépendance des prises en charge des validations ('This is my restaurant, therefore nobody tells me what to do!').

4 En (3), le même glissement sémantique de la coordination à la subordination semble s'opérer avec $\mathrm{OR}$ :

(3) You lay off her, you bastard, or you're dead! (Auster, The Brooklyn, 121) 
Où les validations des prédications coordonnées sont posées comme inversement dépendantes.

6 A cette apparente variabilité opératoire des marqueurs de coordination s'ajoute une variabilité sémantique bien connue elle aussi : au sein même d'un fonctionnement de hiérarchie conceptuelle par exemple, AND peut construire une relation de successivité temporelle, de concomitance, de conséquence, etc.

7 Cette double variabilité pose au linguiste qui s'y intéresse de sérieux problèmes théoriques (Gournay 2006 parle de 'casse-tête', et l'on voit apparaitre sur ce sujet les adjectifs 'tricky' ou 'tough' sous la plume de nombreux linguistes, dont Blakemore \& Carston (2005), pour deux raisons principales. D'une part, elle fait obstacle à toute tentative de définition unifiée des différentes dimensions (syntaxique, sémantique, discursive) des coordonnants, suggérant qu'elles doivent être analysées de façon séparée (cf. Blühdorn 2008). Or c'est bien dans l'interface entre ces différentes dimensions de la langue que nait le sens, et il est donc intéressant de considérer justement leurs points d'interaction. D'autre part, cette double variabilité pose la question de l'arbitraire des relations forme/opération (polyvalence) et forme/sens (polysémie). La recherche des opérations invariantes des marqueurs linguistiques, et de ce que l'on peut nommer 'formes schématiques', sorte de génomes des opérateurs, semble exclure logiquement toute notion de polyvalence, et encore plus de polysémie. Pourtant, comme c'est le cas avec bien d'autres morphèmes (les prépositions par exemple), la polyvalence des coordonnants est un phénomène indubitable, et c'est précisément cette double variabilité (polyvalence et polysémie), qui nous intéresse ici, tout autant que leurs formes schématiques. Quand et pourquoi un coordonnant syntaxique instruit-il une représentation/interprétation de dépendance sémantique? Cette variabilité est-elle inscrite dans ses gènes, dans sa forme schématique? Quelles opérations relèvent d'une forme schématique ou de son interaction avec des paramètres contextuels? Enfin et surtout : peut-on délimiter/formaliser, et intégrer à une forme schématique (invariante) un principe de variance ou, (comme proposé dans Sekali 1992 pour les subordonnants polyvalents SINCE et FOR), un schéma intégré de déformabilité ?

\section{Objectif et démarche}

8 L'objectif de cette étude est d'exemplifier, à travers une analyse des coordonnants anglais AND et OR, l'interaction privilégiée entre les formes schématiques des marqueurs et les instructions de cible qu'apportent les autres marqueurs de construction de l'énoncé complexe. Notre hypothèse ici est que le type de lien conceptuel (hiérarchisé ou non) construit par les marqueurs de coordination, dépend d'un certain nombre d'instructions contextuelles qui sélectionnent, non pas directement la valeur sémantique de la relation, mais la cible (ou portée) de la relation. Dans cette optique, l'opération de coordination, intrinsèquement souple et polyvalente, se fixe alors selon la cible convoquée, et en particulier selon le niveau de structuration des énoncés coordonnés sur lequel porte l'opération de lien: niveau prédicatif ou énonciatif, et la sélection d'un niveau cible de portée des opérations de connexion est elle-même instruite par un faisceau de relations notionnelles (lexicales) et de déterminations contextuelles grammaticales qui, en interaction avec les formes schématiques des coordonnants, définissent un certain paramétrage du sens. 
9 Si l'on prend comme point de départ les marqueurs et non les structures, les trois dimensions de l'analyse cités plus haut peuvent être pris en compte dans la recherche des formes schématiques dans le cadre des opérations énonciatives: chaque coordonnant peut être reconnu dans son mode opératoire au niveau syntaxique, sémantique et pragmatique (ou discursif), et c'est précisément l'interface entre ces trois niveaux d'analyse qui permet de distinguer les formes schématiques propres à chaque marqueur. Nous proposons donc, dans un premier temps, de rappeler brièvement les formes schématiques dégagées dans nos travaux précédents pour AND et $\mathrm{OR}$, puis d'analyser les paramètres contextuels qui définissent une cible prédicative ou énonciative de l'opération de coordination.

\section{AND/OR : formes schématiques et ciblage du niveau prédicatif des énoncés coordonnés}

10 Nous envisageons la coordination en tant qu'opération d'assertion d'un lien, sur la base d'un point d'anaphore (nbp1 : ce concept, associé à la coordination, est défini plus bas). Par la coordination, plusieurs notions, ou plusieurs relations prédicatives elles-mêmes plus ou moins déterminées et assertées, sont nouées, scellées ensemble autrement que par une simple juxtaposition ordonnée, pour former une macro-notion, ou un macroénoncé. (nbp2: on s'intéressera ici essentiellement à la coordination interpropositionnelle, mais l'analyse s'applique de la même manière aux macro-notions (cf. Gournay 2007).

11 L'assertion inter-propositionnelle marquée par le coordonnant est posée et prise en charge par un énonciateur, de sorte que le coordonnant prend non seulement une fonction de lien prédicatif, mais définit également la modalité de ce lien. Il s'agit donc d'asserter un lien prédicatif entre deux constituants ou plus, (une sorte de métaassertion), dont la modalité et la relation à la chaîne linéaire seront différentes selon le coordonnant utilisé. Trois dimensions essentielles définissent ainsi l'opération de coordination :

Le marquage d'un lien prédicatif et énonciatif dynamique et orienté entre plusieurs entités ;

13 Un rapport étroit mais différencié selon le marqueur, entre cette dynamique prédicative et la dynamique de la chaîne linéaire (c'est d'ailleurs là le trait le plus saillant, et peutêtre définitoire, de l'opération de coordination);

Une distribution particulière de l'information au sein de l'ensemble formé.

15 Les trois dimensions sont bien entendu interdépendantes au niveau même de la forme schématique : la relation qu'entretient un coordonnant avec la dynamique linéaire et avec l'organisation thématique des informations, par exemple, spécifie la structure syntaxique, qui à son tour instruit l'action interprétative.

\subsection{AND}

16 En tant qu'opérateur d'assertion de lien, AND marque un ordonnancement prédicatif iconique, c'est-à-dire une avancée prédicative qui suit l'ordre linéaire (Crake-Rossette 2003 parle de construction ascendante, qu'elle oppose à une construction en boucle) : le deuxième élément coordonné constitue un ajout prédicatif et modal (qualitatif) sur le 
premier et les éléments, à l'issue de la coordination en AND, sont posés comme sémantiquement liés et pragmatiquement co-orientés. Considérons par exemple l'extrait suivant:

(4) 'No sign of her. She's simply forgotten.'(...)

' It's all so unlucky my grandfather giving up his drive, and mother backing out of her hospital meeting, and having all the committee down on her. And Henley: I'd even coaxed Henley away from his bridge! He escaped again just before you came. Undine promised she'd have the boy here at four. It's not as if it had never happened before. She's always breaking her engagements.' (Wharton, Custom, 97)

Dans cet extrait la narratrice/personnage exprime son exaspération devant l'absence d'une Undine très attendue. La coordination par AND de trois subordonnées gérondives extraposées à fonction sujet d'un même prédicat recteur 'be unlucky', dispose les événements dans un ordre croissant de 'unluckiness' au fil du discours. Si les trois gérondives partagent le même prédicat, et sont rassemblées par 'all', leur position dans l'ordre linéaire est imposée par AND comme iconique d'une surenchère qualitative sur la notion 'unlucky', qui constitue ici le point d'anaphore notionnel sur lequel elles s'enchaînent et s'accumulent. Les événements 'giving' up his drive', 'backing out of her hospital meeting', et 'having all the committee down on her' ne sont pas représentés dans une successivité temporelle, ni même dans une concomitance temporelle: la forme ING définit ici plutôt une concomitance modale, ou qualitative. Le coordonnant qui les rassemble inscrit ces événements dans un mouvement prédicatif où un événement devient le point d'ancrage d'une avancée qualitative et discursive dans le suivant. Le dernier AND est précédé d'un point, dont le rôle est de clore l'énumération, or le coordonnant vient ici contredire cette clôture, en reprenant l'énoncé précédent (et même l'ensemble de la séquence), pour continuer dans le même sens et aller encore plus loin. AND asserte ainsi un ajout prédicatif, que l'on pourrait gloser par 'attention, cela ne s'arrête pas là, j'ai encore mieux!' L'adverbe 'even' dans 'I'd even coaxed Henley away from his bridge !' confirme le rattachement inattendu mais avéré de l'événement 'coax Henley away from his bridge' par AND à la série précédente, et pourrait être glosé par 'et pour couronner le tout..'.

On retrouve ce mouvement prédicatif dans l'énoncé suivant :

(5): "I'm thirty years old," he told his new boss, "and forty pounds overweight. I haven't slept with a woman in over a year, and for the past twelve mornings i've dreamt about traffic jams in twelve different parts of the city. I could be wrong, but I think I'm ready for a change." (Auster, Brooklyn, 207)

AND, l'anti-restriction, va encore plus loin, rassemble des unités même hétérogènes dans une visée énonciative commune. La paraphrase possible du coordonnant par 'furthermore' traduit d'ailleurs assez bien ce mouvement à la fois linéaire (further) et modal (more).

Dans l'énumération suivante:

(6) Things break all the time. Glass, and dishes, and fingernails. Cars and contracts and potato chips. You can break a record, a horse, a dollar. You can break the ice.

There are coffee breaks and lunch breaks and prison breaks. (COCA)

l'hétérogénéité sémantique des items reliés est flagrante, mais le coordonnant les relie sur le point d'anaphore notionnel 'break', les co-oriente dans une progression linéaire à la fois quantitative et qualitative qui renforce l'assertion première (things break all the time). 
AND impose ainsi une continuation du discours et une progression prédicative qui sont solidaires de la dynamique linéaire: la prédication relationnelle va dans le même sens que la chaine linéaire, et impose même un mouvement en avant, ici qualitatif, ou modal.

Figure 1 : Mouvement prédicatif marqué par AND

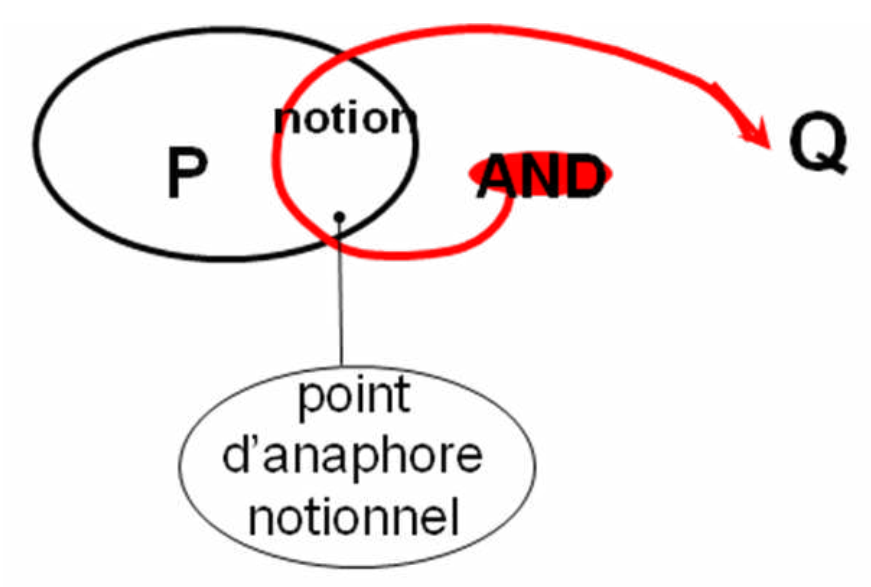

further more

\section{dynamique discursive}

La progression prédicative est toujours assertée, avec AND, sur la base d'un point d'intersection, ou point d'anaphore, enjeu du lien de coordination. Ce sont, dans ces exemples, des notions prédicatives dans $\mathrm{P}$ qui sont la cible d'une reprise pour qualification au sein de l'énoncé coordonné, avec une intersection des domaines notionnels de $\mathrm{P}$ et $\mathrm{Q}$. Le point d'anaphore est ici une notion à qualifier. Ce mouvement d'avancée prédicative et modale sur la base d'un point d'anaphore (Cf. Fig.1) est quasiment paraphrasé par 'What's more' dans l'exemple suivant, qui met en emphase l'intensification qualitative issue de la coordination avec AND :

(7) "His voice", she said bitterly, "is a Christian voice, and what's more it's

Presbyterian." (Saroyan, Aram, 89)

Bien que les Presbytériens soient une sous-classe des Chrétiens, le fait d'avoir une voix presbytérienne est représenté ici, du fait de la coordination en AND, comme encore plus désagréable (cf. l'adverbe 'bitterly') qu'une voix simplement chrétienne.

Dans cet ordre prédicatif asserté par AND dans la structure P AND Q, P sert de base à une avancée prédicative, qui sera qualitative ou quantitative selon le point d'anaphore.

\subsection{OR}

Le coordonnant OR effectue également un lien entre deux ou plusieurs notions ou énoncés, mais le rapport entre l'ordre prédicatif qu'il marque et la dynamique linéaire n'est pas du tout le même : avec OR, les éléments sont joints de façon à la fois verticale et horizontale ; la maille du tricot discursif en est donc différente. OR, on le sait, coordonne un élément à son autre, de sorte que dans la construction $\mathrm{P} O \mathrm{R} Q$, le coordonnant revient sur le domaine notionnel de $\mathrm{P}$ pour l'ouvrir à son extérieur, et coordonne $\mathrm{P}$ ' à la représentation d'autres possibles (cf. Fig.2): 
Soit $\mathrm{Q}$ est directement « l'autre de $\mathrm{P}$ » (son complémentaire), ce qui est finalement assez rare, avec des exemples du type 'Stand up or sit down', 'To be or not to be, that is the question'.. et l'on construit alors simplement l'ouverture d'une alternative, ce qui permet des opérations de parcours de l'intérieur et de l'extérieur de la notion complexe envisagée $(\mathrm{P} /$ nonP) ;

Soit Q est « un autre que $\mathrm{P}$ », coordonné à la branche P' préalablement ouverte par le coordonnant, du type 'Your money or your life !'

Figure 2 : Mouvement prédicatif marqué par $\mathrm{OR}$
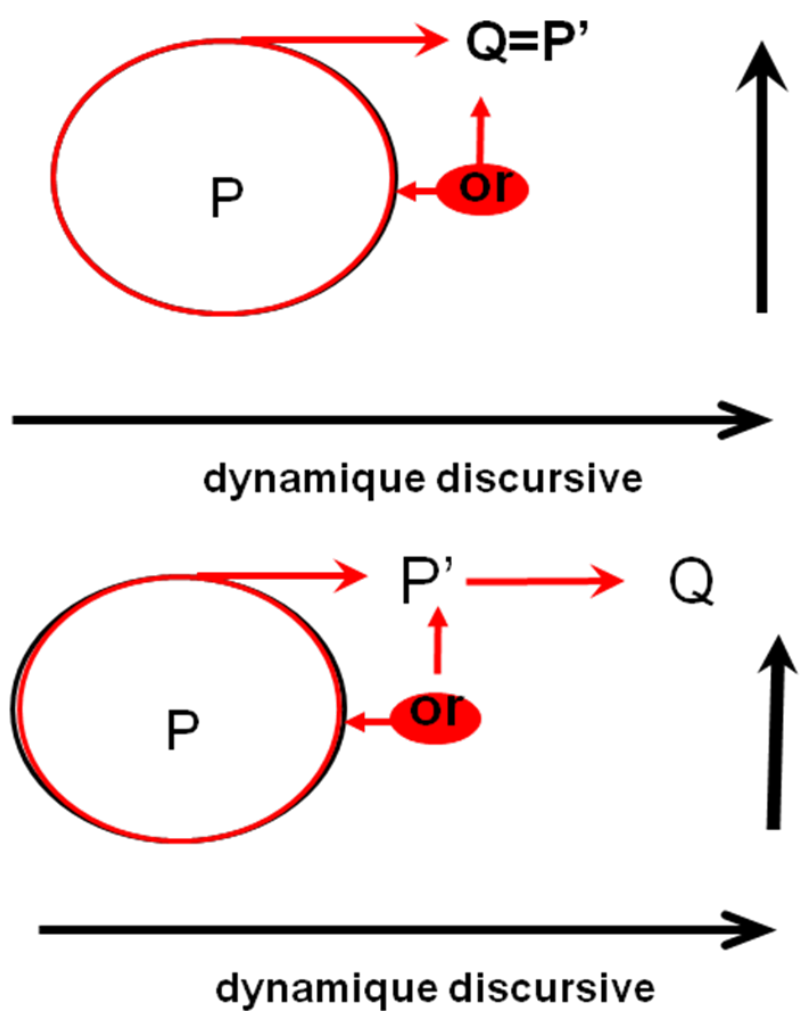

On a ainsi avec OR, un mouvement prédicatif différent de celui marqué par AND, linéaire aussi mais d'abord vertical, qui réévalue $P$ pour ouvrir sur un choix. Ce choix est marqué comme possible ou contraint selon les déterminations internes: associé à des questions fermées ou des modalités impératives, par exemple, OR ouvre l'alternative $\mathrm{P} / \mathrm{P}$ ' et ce sont les modalités assertives dans $P$ et $Q$ qui définissent un appel au co-énonciateur, et une injonction de choix exclusif. Dans l'énoncé suivant par exemple:

(8) "Raymond, do you want to stay with your brother Charlie here in Los Angeles or do you want to go back to Wallbrook?"

"Go back to Wallbrook and stay with my brother Charlie."

"No, there are two separate things, then you make that choice: the one or the other." (Levinson, Rain Man.)

L'énonciateur, par une question fermée, impose à Raymond un choix binaire entre deux prédicats complémentaires : rester ou partir. Il y a ouverture sur un choix restreint, et injonction de choix. Raymond bloque la stratégie de l'énonciateur en refusant de choisir et en associant les deux prédicats par AND. Cette ouverture sur le complémentaire de $\mathrm{P}$, avec balayage des deux valeurs $\mathrm{P} /$ non $\mathrm{P}$, est également représentée dans sa complexité dans l'exemple (8), où l'on observera le jeu subtil que les coordonnants OR et BUT autorisent sur la définition des notions prédicatives coordonnées : 
(9) "You must have been too drunk to notice."

"Drunk or not, there's one thing I have to know. Did you mean it about wanting to get out of the city - or was it just talk?"

"I meant it, but it was also just talk."

"It can't be both. It has to be one or the other."

"I meant it, but I also know it will never happen. Therefore, it was just talk."

(Auster, Brooklyn, 206.)

Dans les exemples (10) à (12) en revanche, Q n'est pas le complémentaire direct de $P$, mais est coordonné à ce complémentaire ouvert par $\mathrm{OR}$, ou à un autre que $\mathrm{P}$ :

(10) Shades drawn, his body enveloped in the womblike dark of his tiny home, he would sleep until his eyes opened of their own accord- or, as often happened, a noise from somewhere in the building jolted him awake. (Auster, Brooklyn, 132)

(11) I mostly blame myself, but Tom was old enough to have taken the initiative, and he could have sent me a word whenever he'd chosen to. Or, if not me, then his first cousin, Rachel, who was also in the Midwest at the time, doing her postgraduate work in Chicago. (Auster, Brooklyn, 18)

(12) Tom finally understood that he didn't have it in him to finish. Or, if he did have it in him, he couldn't persuade himself to believe in the value of doing it anymore. So he left Ann Arbor and returned to New York, a twenty-eight-year-old has-been without a clue as to where he was headed or what turn his life was about to take. (Auster, Brooklyn, 19)

Bien souvent d'ailleurs, cette ouverture verticale de la notion de $\mathrm{P}$ à son extérieur (non $\mathrm{P}$ ou autre que P), est explicitée dans les énoncés avec un 'if not', comme dans les exemples (11) et (12), qui retracent le mouvement complexe, vertical puis horizontal, marqué par le coordonnant $\mathrm{OR}$.

Les deux coordonnants définissent ainsi des mouvements prédicatifs qui n'ont pas le même rapport à la dynamique linéaire: AND reprend un élément de $\mathrm{P}$ qui se trouve renforcé ou avancé, par la prédication de $\mathrm{Q}$. OR quant à lui reprend l'ensemble $\mathrm{P}$ pour l'associer à son autre, ou à un autre, mettant en regard plusieurs possibles référentiels. AND asserte ainsi un mouvement prédicatif linéaire prospectif; OR marque d'abord un arrêt, une interruption du discours pour ouvrir le champ des prédications possibles. C'est là aussi une dimension linéaire, mais d'abord verticale, par superposition plutôt que par progression, qui donne une deuxième dimension au texte ou au discours. OR permet ainsi de désasserter P dans la coordination, de l'envisager comme non validé ou non validable, ce que ne permet pas AND.

Les formes schématiques des deux coordonnants AND et OR, telles que brièvement décrites ici (nbp 3 : pour une analyse plus détaillée, et une comparaison avec BUT et FOR, voir Sekali 2007, 2010a. et 2010b), en prenant en compte différentes dimensions évoquées plus haut, font ressortir pour les deux marqueurs la trace d'une opération d'assertion d'un mouvement prédicatif inter-propositionnel ou inter-syntagmes orienté, qui est ancré dans un rapport étroit avec la dynamique linéaire du discours, et ne peut s'y soustraire, mais avec des orientations différentes, et des modes d'association propres. 


\section{De la coordination syntaxique à la subordination sémantique : le niveau énonciatif pris comme cible de l'opération de lien} cible (ou portée), laquelle est instruite par une série de paramètres extérieurs au marqueur. Pour ce qui concerne les énoncés complexes, et les marqueurs de relation, un grand nombre de variables contextuelles concourent et interagissent dans la convocation d'une cible (ici nommée 'point d'anaphore' dans l'analyse de AND) ; en particulier :

- Le lexique, les phénomènes d'aspect lexical et de présuppositions (relations notionnelles complexes);

- La détermination grammaticale des noms et des verbes dans les propositions liées ;

- Les reprises, anaphore et deixis ;

- Les schémas intonatifs à l'oral ;

- Les modalités assertives et intersubjectives;

- La relation à la situation de l'énonciation, références situationnelles, situation de discours, interlocution.

Il est important de préciser que ces variables contextuelles ou situationnelles ne fournissent pas directement des instructions de sens, mais plutôt des instructions de cible, c'est-à-dire des instructions sur le niveau de portée des opérations de coordination dans la construction de l'énoncé complexe : niveau prédicatif ou énonciatif. Ainsi, les relations de symétrie/asymétrie, homogénéité/non homogénéité ne sont pas forcément déjà inscrites directement dans les formes schématiques des marqueurs, mais peuvent faire partie des variables 'ouvertes' de la forme schématique, variables opérationnelles plutôt que variables sémantiques.

Nous avons cité plus haut des exemples dans lesquels les coordonnants AND et OR opéraient la reprise d'une notion (nominale ou verbale) pour l'inscrire dans une dynamique de renforcement quantitatif ou qualitatif (AND), ou d'ouverture verticale du domaine notionnel (OR). Dans ces énoncés, les deux coordonnants marquent une opération de lien qui porte sur le niveau prédicatif des énoncés liés, et construit des relations notionnelles. Ces relations ne sont pas hiérarchisées, au sens où elles ne définissent pas de dépendance conceptuelle ni de repérage asymétrique. Les éléments joints sont donc coordonnés du point de vue syntaxique comme sémantique : la relation à la linéarité est modale, en tant qu'avancée prédicative non hiérarchisée, mais la macronotion issue de la coordination retravaille chacune des notions jointes dans la construction complexe.

Outre la reprise d'une notion au niveau prédicatif de l'énoncé $\mathrm{P}$, la coordination peut cibler le niveau énonciatif de la structuration du premier énoncé, c'est-à-dire prendre comme point d'anaphore les repérages de sa validation par rapport aux coordonnées énonciatives ( $\mathrm{T}$ et $\mathrm{S}$ ) ou sa modalité assertive. Dans ce cas, c'est la localisation temporelle de l'énoncé $\mathrm{P}$, ou bien sa prise en charge subjective, qui seront définies comme point d'anaphore, et repères d'une avancée/ouverture soit temporelle, soit modale, de la validation de l'énoncé $Q$.

Ainsi dans les récits au prétérit, comme dans les énoncés (12), (13) et (14), où les prétérits sont des formes aoristiques détachées de tout point de vue subjectif mais localisées

Corela, HS-10 | 2012 
temporellement, les événements coordonnés par AND sont placés dans une successivité temporelle qui suit toujours le mouvement linéaire : le temps des événements suit l'ordre de la prédication. AND pose alors un énoncé comme repère d'une avancée temporelle dans l'autre :

(12) Julie closed $\left(\mathrm{T}_{1}\right)$ the door again and slid $\left(\mathrm{T}_{2}\right)$ the chain into place. (BNC)

(13) Ginyard turned $\left(T_{1}\right)$ and looked down the street $\left(T_{2}\right)$ and spoke $\left(T_{3}\right)$ into the wind. (COCA)

(14) They jammed $\left(\mathrm{T}_{1}\right)$ their hands deep into the pockets of their matching trench coats and stomped away $\left(\mathrm{T}_{2}\right)$. Kyle unlocked $\left(\mathrm{T}_{4}\right)$ his Jeep and got inside $\left(\mathrm{T}_{5}\right)$. He started $\left(\mathrm{T}_{6}\right)$ the engine, turned $\left(\mathrm{T}_{7}\right)$ the heat on high, and thought $\left(\mathrm{T}_{8}\right)$ about driving away. (COCA)

Notons dans ces exemples, que la sélection d'un point d'anaphore temporel, et donc le ciblage énonciatif de la coordination, sont instruits par un ensemble de paramètres contextuels. L'aspect lexical des verbes, de type processus dynamique, parfois même inchoatif, la détermination des verbes au prétérit simple dans un contexte de récit, définissent des événements ponctuels et non des propriétés des sujets. Ce paramétrage semble convoquer une portée particulière de la coordination, et la diriger sur le niveau énonciatif (et non plus purement prédicatif) de la structuration des énoncés joints. C'est le repère $\mathrm{T}$ de la détermination des prédicats qui est ici ciblé, et devient l'enjeu d'une progression linéaire marquée par le coordonnant AND, ce qui construit une relation de consécution. Dans l'extrait (14), qui comprend huit événements au prétérit simple, mais pas tous coordonnés par AND, on peut s'interroger sur la différence de relation instaurée par la coordination (entre T4 et T5 par exemple : 'Kyle unlocked his Jeep and got inside'), et celle définie par la parataxe asyndétique (entre T5 et T6 par exemple: '(Kyle) got inside. He started the engine'). La seule présence d'un point garantit en effet la successivité des événements au prétérit dans une chaîne linéaire déjà orientée. Outre la nécessité rythmique d'une pause marquée par la ponctuation, nous pensons que l'organisation des informations et la stratégie narrative diffèrent. Les événements consécutifs liés avec AND sont ici narrativement co-orientés. La récurrence des verbes d'aspect lexical inchoatif (unlock, get, start) marque déjà des relations inter-événements relayées par AND, de sorte que, par exemple, dans 'Kyle unlocked his Jeep and got inside', le coordonnant prolonge la visée inchoative du premier conjoint dans le deuxième, et pourrait éventuellement être traduit en français par 'pour'. L'ensemble conjoint forme une unité textuelle. Le point et la reprise pronominale de Kyle par 'he' clôturent cet ensemble pour ouvrir ensuite sur une autre série d'événements conjoints et représentés comme une reprise de la dynamique après une brève hésitation du personnage.

Le coordonnant AND s'appuie donc à la fois sur l'aspect lexical et sur les déterminations grammaticales internes aux énoncés liés, pour définir dans ces exemples une cible énonciative de l'assertion d'un mouvement prédicatif ordonné de $\mathrm{P}$ à $\mathrm{Q}$, qui suit et poursuit la dynamique horizontale de la chaîne linéaire sur la base d'un point d'anaphore énonciatif. Ce ciblage énonciatif de la coordination induit un repérage inter-énoncé et donc une asymétrie conceptuelle, l'un des conjoints devenant matrice temporelle de l'autre.

Considérons les instructions de portée de la coordination dans l'énoncé (15) :

(15) She wrote under the name Florence Flowers, a nom de plume from her own mother's maiden name, and she had enjoyed immense success. She was sixty-two years old and had lived a storybook life. (COCA) 
41 Cet exemple est intéressant à plusieurs titres. Les prétérits simples sur 'write' et 'be' ne définissent pas ici des événements ponctuels mais des propriétés de 'she' (écrivain de 62 ans), dans un récit où le narrateur (la fille de 'she'), raconte et explique le caractère de sa mère. Deux énoncés complexes se succèdent ici sur le même schéma : P (prétérit) AND Q ( past perfect), séparés par un point. Remplacer ce point par un nouveau coordonnant AND semble très improbable :

$\left(15^{\prime}\right) ? ?^{*}$ She wrote under the name Florence Flowers, a nom de plume from her own mother's maiden name, and she had enjoyed immense success, and she was sixtytwo years old and had lived a storybook life.

C'est que les propositions ne sont pas liées par AND simplement de façon cumulative. Du point de vue des repérages internes aux énoncés joints, on note que les formes aspectuelles de past perfect dans les énoncés Q ('she had enjoyed immense success' et 'had lived a storybook life'), construisent à chaque fois une évaluation rétrospective, qui prend $P$ ('she wrote..' ; 'she was sixty-two years old') comme thème et point de vue repère. Dans ce contexte, l'avancée prédicative marquée par AND est à la fois notionnelle et énonciative, et la coordination porte sur la détermination prétérite ciblée en tant que point d'anaphore temporel et subjectif (repère temporel d'un bilan rétrospectif et repère subjectif d'une évaluation qualitative). Ensemble, le coordonnant, les déterminations verbales et l'orientation dynamique du texte, construisent une organisation particulière de l'information et une progression du fait vers son évaluation. Les deux énoncés complexes, séparés par un point, ne pourraient être coordonnés par AND, non seulement pour des raisons rythmiques, mais parce que les deux sous-ensembles ne forment pas ici un ensemble qui soit organisé dans cette répartition anaphorique de l'information et des repérages.

Dans les exemples (16) à (19) suivants, AND marque toujours une avancée prédicative parallèle à la chaîne linéaire, mais cette avancée prend le sens d'une valeur argumentative de conséquence :

(16) Ed Donnelly is still bothered by a side injury and will miss his starting turn. ( BROWNS)

(17) This is my restaurant, and nobody tells me what to do ! (Auster, Brooklyn, 122)

(18) I've always found that torturing women has bad side effects. For some reason, it's regarded as particularly heinous and rouses opinion against you. (COCA)

(19) “They like being in people's homes, having that one-on-one relationship," Stone says. A perennial complaint from aides in facilities is that they're responsible for too many residents and can barely manage to complete their assigned tasks, let alone have meaningful conversations. (Washington Post, 10/05/09)

On note ici la récurrence des présents simples, qui construisent ici des propriétés du sujet et non des événements ponctuels; les validations des relations prédicatives de part et d'autre du coordonnant sont corrélées par AND, l'une servant de repère de la prise en charge modale de l'autre. Dans ces exemples, Q est défini comme la conséquente évidente de $P$, relevant de la logique interne (cf. will en (16)). Le modal WILL inscrit dans l'énoncé $Q$ peut d'ailleurs être considéré comme anaphorique du lien de coordination lui-même, et porte donc sur l'assertion inter-propositionnelle plutôt que sur l'assertion intrapropositionnelle, comme nous le développerons plus loin.

Dans cette configuration, AND explicite un lien de repérage inter-propositionnel qui cible aussi le niveau énonciatif des énoncés joints, les deux énoncés devenant inséparables à partir, cette fois, du point d'anaphore S. Ainsi, lorsque le point d'anaphore de cette progression prédicative n'est plus la coordonnée T mais $S$, c'est-à-dire la prise en charge 
de sa validation par une instance subjective, on entre dans l'argumentation: $\mathrm{P}$ est le repère d'une prise en charge de $Q$ dans un mouvement d'importation des validations et de leur prise en charge d'un énoncé vers le suivant.

Bien entendu, cette cible particulière de la coordination, qui construit ce sens relationnel de conséquence, n'est pas toujours, ni uniquement, instruite par la détermination verbale au présent simple. Les paramètres contextuels qui convoquent cette cible sont nombreux et complexes. Dans l'énoncé suivant par exemple:

(20) The week before Thanksgiving, Laney began to have trouble breathing and spent a few days in the hospital ; she came home with an oxygen tank. (Washington Post, 10/05/09)

Les prédicats sont déterminés au prétérit, représentent des événements ponctuels, et pourtant le lien marqués par AND est interprétable non seulement comme un lien de consécution, mais aussi de conséquence, probablement en raison du lien d'inférence thématique qui existe au niveau lexical entre 'have trouble breathing' et 'go to hospital'. L'aspect lexical inchoatif de 'begin' a également une incidence sur la valeur résultative du prédicat 'spend', le tout renforcé par le mouvement linéaire de co-orientation des conjoints marqué par le coordonnant. Il s'agit donc, comme toujours en langue, d'un paramétrage multi-dimensionnel, dont nous tentons ici de formaliser quelques paradigmes. En (21):

(21) Rozzie Laney, the 91-year-old woman propped up in her hospital-style bed on this Monday afternoon, didn't respond. She'd had Alzheimer's disease for many years and no longer spoke. (Washington Post, 10/05/09)

Le past perfect sur le prédicat 'have Alzheimer's disease' est plus clairement constitutif d'un état résultant ('no longer spoke') dont il définit l'origine temporelle et modale. L'aspect ici semble bien convoquer une cible énonciative de la coordination, qui, linéairement contrainte, instruit à son tour une relation de conséquence.

Ainsi, lorsque la coordination en AND prend pour cible le niveau énonciatif de la structuration des énoncés qu'il relie, on peut parler d'un phénomène de subordination sémantique, malgré la coordination syntaxique, dans le sens où l'un des énoncés est défini comme repère du calcul de la validation d'un autre, dans une position sémantiquement hiérarchisée. Dans Sekali (sous presse), nous avons proposé de définir la subordination sémantique en tant qu'opération linguistique marquée, par laquelle un énonciateur asserte, par un ou plusieurs signes linguistiques en interaction, l'existence d'un processus de repérage inter-énoncés qui prend pour cible le niveau énonciatif de la structuration du premier conjoint $\mathrm{P}$.

Cette définition permet d'unifier l'analyse à l'interface entre les dimensions syntaxique et sémantique des opérations de lien. La subordination sémantique (ou asymétrie conceptuelle) peut ainsi concerner tous les énoncés complexes, quelle qu'en soit la structure syntaxique, dès lors qu'un énoncé est défini comme matrice et repère de la prise en charge subjective et/ou de la localisation temporelle d'un autre qui lui est subordonné. Avec AND on peut alors parler de coordination syntaxique mais de subordination sémantique chaque fois que la cible de l'opération est le niveau énonciatif du premier conjoint: coordonnées énonciatives $S$ ou $T$, ou modalités assertives, ce qui définit une hiérarchie et une dépendance des validations coordonnées (et induit d'ailleurs systématiquement la non-symétrie linéaire des propositions).

51 Si la convocation de cette cible de l'assertion inter-propositionnelle est instruite par des paramètres et variables contextuelles et situationnelles, la complexité reste de pouvoir 
identifier ces paramètres, et éventuellement de les formaliser en paradigmes, dans le but de tenter de retracer la construction du sens relationnel interprété. Notre observation de différents corpus (British National Corpus écrit et oral, Browns American Corpus, Corpus of Contemporary American English, ainsi qu'un panel de romans britanniques et américains) a pu révéler quelques récurrences intéressantes, suggérant des paradigmes de paramètres pertinents. Dans la sélection d'une cible au niveau énonciatif, convoquant un lien de subordination sémantique avec le coordonnant AND, le paradigme de la détermination grammaticale des noms et verbes dans les propositions liées, et des modalités assertives, est logiquement prépondérant (même s'il interagit toujours avec d'autres paradigmes). Le tableau ci-dessous (très incomplet) donne néanmoins une tendance claire concernant la relation entre la détermination verbale (associée à l'aspect lexical des prédicats dans les propositions liées), le type de lien inter-propositionnel convoqué, et quelques sens relationnels construits :

Tableau 1

\begin{tabular}{|l|l|l|}
\hline \multicolumn{2}{|l|}{ Instructions de subordination sémantique et valeurs relationnelles avec AND } \\
\hline Valeur relationnelle & Détermination verbale en P & Détermination verbale en Q \\
\hline Consécution & $\begin{array}{l}\text { Prétérits ponctuels } \\
\text { Présent ponctuels } \\
\text { EVENEMENTS }\end{array}$ & $\begin{array}{l}\text { Prétérits ponctuels } \\
\text { Présent ponctuels } \\
\text { EVENEMENTS }\end{array}$ \\
\hline Conséquence & $\begin{array}{l}\text { Présent sur verbes dynamiques } \\
\text { PROPRIETES } \\
\text { Aspect Past perfect } \\
\text { EVENTS }\end{array}$ & $\begin{array}{l}\text { Présents sur verbes dynamiques } \\
\text { PROPRIETES } \\
\text { Prétérits statifs/ Formes en TO } \\
\text { ETATS RESULTANTS/ INTENTIONS }\end{array}$ \\
\hline Concomitance & $\begin{array}{l}\text { Aspects Be-ing } \\
\text { POINT DE VUE SPECIFIQUE } \\
\text { Présents sur verbes statifs statifs } \\
\text { ETATS }\end{array}$ & $\begin{array}{l}\text { POINT DE VUE SPECIFIQUE } \\
\text { Présents sur verbes statifs } \\
\text { Prétérits statifs } \\
\text { ETATS }\end{array}$ \\
\hline
\end{tabular}

Les aspects lexicaux, ainsi que les temps et aspects grammaticaux au niveau des repérages des validations semblent ainsi offrir un paradigme de variabilité essentiel dans la structuration du lien marqué par la coordination par AND, en instruisant une cible particulière de l'opération marquée par le coordonnant.

\section{Ciblage de la modalité assertive des conjoints : le cas des coordonnées 'pseudo-impératives'}

Il est des cas où le coordonnant cible la modalité assertive même de $\mathrm{P}$ dans la construction du lien. Les énoncés coordonnés dits 'pseudo-impératifs', qu'on appelle aussi 
'impératifs conditionnels', sont intéressants à cet égard, par la forme assertive très particulière qui est inscrite dans $P$, et par le fait qu'ils déclenchent quasisystématiquement une interprétation de subordination sémantique. On y observe même un phénomène de report assertif, au sens où moins l'assertion intra-propositionnelle est marquée, plus l'assertion inter-propositionnelle définie par le coordonnant sera forte.

Voici quelques exemples avec AND et OR.

(22) "Play it smart, and you'll have money." (Kubrick, Killing)

(23) "Just call me tonight or I go crazy !" (Lumet, Q\&A)

Dans ces énoncés, nous observons un paradigme commun : la validation effective du premier conjoint (play it smart, call me tonight) n'est pas marquée comme assertée, les relations prédicatives ne sont que validables, et non validées. Ce type de modalité assertive dans $\mathrm{P}$ déclenche une cible énonciative de la coordination, et donc un lien de repérage inter-énoncé hiérarchisé au niveau du calcul des validations et de leur prise en charge modale.

\section{1. 'Pseudo impératifs' avec AND}

Dans les énoncés dits 'impératifs conditionnels' avec AND, ni P ni Q ne sont, en réalité, assertés. Considérons les énoncés suivants:

(24) "Trust me darling and you will be all right. (Ashford, Love, 42)"

(25) "Give us the contents of this room, and we'll consider the account square."

(Auster, Brooklyn, 207)

(26) "Try to rise above your station in this house, and you will find yourself back in

Ireland !" (Film: Huston, D., Mr North, 1988)

(27) "You put me back in jail and I'm finished. I'm a dead man." (Auster, Brooklyn,

(28) You get on that mule and I kill you. (Brooks, Hunt)

(29) A minute more and he would have missed the train. (Quirk \& al, CGEL, § 11.53.)

(30) Stop major! Another step and I fire! (Sturges, Coat)

L'impératif est une modalité inter-subjective déontique avec injonction par l'énonciateur de validation de P par le co-énonciateur. Les énoncés (24) et (25) ci-dessus pourraient certes être interprétés comme des injonctions ('trust me', 'give us the contents of this room'), mais certainement pas les énoncés (26) et (27) ('Try to rise above your station in this house', 'You put me back in jail'). Comme le remarque Franke (2008), dans ces énoncés coordonnés par AND, la valeur déontique ou non de la modalité assertive en P est en réalité intimement liée à l'aspect désirable ou non de la validation de $Q$ pour le coénonciateur, ce qui explique la valeur de menace en (26). Que P soit une proposition ou un groupe nominal (souvent des prédicats nominalisés, avec des déterminations en 'a' et des comparatifs, comme dans les exemples (29) et (30)), on ne peut en réalité parler d'impératifs dans ces constructions. En effet, contrairement aux pseudo-impératifs en OR, (où il $\mathrm{y}$ a une réelle visée de $\mathrm{P}$ par l'énonciateur, comme nous le développons plus bas), avec AND, la validation du conjoint $P$ n'est ni visée par l'énonciateur, ni prise en charge subjectivement, ni l'objet d'une injonction déontique. Il n'y a finalement, dans ces constructions particulières, aucune marque d'assertion ni même de modalité assertive spécifique du conjoint $\mathrm{P}$, ni d'ailleurs du conjoint $\mathrm{Q}$.

S'il n'y pas de modalité déontique dans $\mathrm{P}$ avec AND, on peut néanmoins parler d'un repérage intersubjectif distancié de l'énonciateur par rapport à l'origine de la validation potentielle de $\mathrm{P}$ et de $\mathrm{Q}$. L'énonciateur reporte sur le co-énonciateur (s'il se reconnait 
comme tel) la validation potentielle de $\mathrm{P}$ (Give us the contents of this room ; Try to rise above your station in this house, You get on that mule) dans son rapport à celle de $Q$ : (we'll consider the account square; you will find yourself back in Ireland; I kill you). La valuation positive ou négative de $\mathrm{P}$, et la prise en charge des énoncés liés, sont laissées à l'appréciation du co-énonciateur, 'en connaissance de cause' : 'if you want $Q$, go ahead and do P!' Ce qui est asserté par l'énonciateur dans ces structures, c'est le lien de repérage inter-énoncés lui-même, le premier énoncé étant imposé comme repère modal du second. En résumé, l'énonciateur construit une image distanciée et autonome du coénonciateur sur la prise en charge des énoncés liés, mais impose le lien $\mathrm{P} / \mathrm{Q}$ par le coordonnant, qui relaie le manque d'assertion intra-énoncé par une assertion interénoncé très forte.

Les paramètres contextuels récurrents dans ces configurations sont encore une fois principalement des marqueurs de détermination verbo-nominale bien particuliers: présents simples sur les verbes, détermination non factive des noms dans $\mathrm{P}$, et, pour ce qui concerne le conjoint $Q$ : présents simples, modalités de type 'will' ou 'would' principalement. Notons également que, dans ces configurations pseudo-impératives en AND, les sujets des deux propositions doivent être explicités, et qu'il est impossible de faire l'ellipse du sujet dans $Q$, même s'il est co-référent à celui de $P$. On a donc une suspension de l'assertion dans $\mathrm{P}$ avec des valeurs de potentiel.

Ces déterminations contextuelles ne sont pas à considérer comme des 'contraintes' dans ces structures, mais plutôt comme des paramètres de sélection d'une cible du coordonnant, qui, dans ce cas, portera sur le niveau des modalités assertives des conjoints : en l'absence d'assertion de $\mathrm{P}$ et de $\mathrm{Q}$, la seule assertion qui soit marquée avec AND est finalement celle du lien inter-propositionnel. AND marque l'assertion et la prise en charge du lien de repérage $\mathrm{P} / \mathrm{Q}$ en tant qu'inéluctable et garanti par l'énonciateur, de sorte que le modal WILL, très souvent présent dans ces énoncés, doit être considéré comme anaphorique de la modalité assertive inter-propositionnelle construite par le lien de coordination.

Le pseudo impératif, ou présent simple, dans ces énoncés, ouvre vers une validation contextuelle, et non situationnelle, comme c'est d'ailleurs le cas lorsqu'il est employé dans les subordonnées en IF et WHEN ('When he comes, I'll show him the garden'). En tant que détermination minimale, le présent simple représente l'image de la relation prédicative sans ancrage temporel de sa validation.

61 Les déterminations modales ou temporo-aspectuelles dans les conjoints $Q$ marquent ainsi des repérages anaphoriques qui sont contextuels plus que situationnels :

(31) Walk through that room, however, open the thickly paned double glass doors at the rear, and you found yourself in an altogether different environment. (Auster, Brooklyn, 122)

(32) Board the train and you enter an exquisite world of intricate marquetry, engraved glass, mosaic floors and brasswork. (BNC)

Les premiers conjoints dans les exemples (31) et (32) invitent ainsi le lecteur (coénonciateur) à une validation imaginaire d'entrer dans le restaurant ou de monter dans le train, afin d'en expérimenter les conséquences, tout aussi imaginaires : ce qui est asserté là encore, c'est un lien de corrélation automatique, systématique, entre les deux validations potentielles, d'où les paraphrases possibles en 'if' ou 'whenever'. Le prétérit sur le verbe find en (31) : 'you found yourself.' embarque le lecteur dans la situation du récit et définit une validation fictive, corrélative à celle des prédicats dans $P$. 
63 Dans tous ces cas, lorsque c'est le niveau de la modalité assertive des énoncés joints qui est ciblé par la coordination, seul le coordonnant, et la subordination sémantique, sont assertés par l'énonciateur. On observe ainsi que plus l'assertion intra-propositionnelle est faible, plus l'assertion inter-propositionnelle est forte et qualitative. Le premier conjoint perd son statut d'énoncé libre pour s'insérer dans une assertion complexe contextuelle. Ce mouvement prédicatif iconique, avec AND, d'assertion d'une relation de repérage inter-propositionnel des modalités assertives, est quasiment paraphrasé dans l'énoncé (33) :

(33) If you're going to choose one of them to lead your people, you'll want the fighter [...] You choose the strong and clever over the weak and kind.'

'That's pretty brutal stuff, Nathan. Take your argument one step further, and the next thing you'll be telling me is that Stalin should be revered as a great man.' (Auster, Brooklyn, 55)

Le modal 'will', anaphorique du lien inter-propositionnel inéluctable marqué par AND, est d'ailleurs associé ici à l'aspect 'be-ing' ('you'll be telling me'), forme de reprise contextuelle anaphorique d'une potentielle validation de 'take your argument further'.

\subsection{AND et IF}

La construction d'un sens relationnel plus ou moins conditionnel est issue d'un certain nombre de paramètres contextuels qui, en interaction avec la forme schématique du relateur, définissent la cible du repérage inter-énoncés, ici un repère assertif de type validation suspendue, potentielle. On ne peut pour autant considérer que ces coordinations avec AND sont équivalentes à une structure conditionnelle en IF.

(33) Take your argument one step further, and the next thing you'll be telling me is that Stalin should be revered as a great man.

(33') If you take your argument one step further, the next thing you'll be telling me is that Stalin should be revered as a great man.

Les travaux en particulier d'Anne Trévise sur l'anglais (cf. en particulier Trévise 1999, et Trévise \& Constant 2007) et de Sarah de Vogüé sur le français (De Vogüé 1999), montrent que le subordonnant construit un repère fictif, un changement de plan par rapport au réel. IF instruit le balayage des deux branches de la bifurcation notionnelle, et la prise en compte de l'occurrence de la protase comme condition d'une validation de l'apodose. La nature de la distanciation par rapport au réel sera marquée par les éléments contextuels, notamment les temps et aspects dans la protase. Avec les conditionnelles en IF, la subordination est donc à la fois syntaxique et sémantique, mais dans un rapport inverse : la subordonnée en syntaxe est la matrice repère en sémantique.

Si l'on retrouve, comme avec AND, une suspension de l'assertion dans la protase, instruite par IF lui même, et déterminée par les marqueurs de repérage internes à la protase, en revanche le mouvement prédicatif n'est pas le même qu'avec AND et les relations intersubjectives non plus. Avec AND, conformément à sa forme schématique, le conjoint $Q$ découle de $\mathrm{P}$ dans l'ordre linéaire comme dans le repérage énonciatif, dans une relation qui relève d'ailleurs plus de la conséquence potentielle que de la condition. Avec IF en revanche, la relation de subordination sémantique n'est plus soumise à la dynamique linéaire, le repère peut être antéposé ou postposé au repéré. Il s'agit là d'un mouvement prédicatif non linéaire, non orienté, avec structuration d'un lien de repérage contextuel sur la base d'un repère fictif de validation de la protase. De plus, le lien inter-énoncés lui- 
même, tel qu'il est défini par IF, n'est pas assorti d'une modalité assertive et intersubjective comme c'est le cas avec AND, mais représenté comme inhérent.

L'énoncé coordonné en (33) comporte plusieurs marqueurs qui, nous l'avons dit, paraphrasent le mouvement prédicatif iconique marqué par 'AND'. La substitution avec IF peut reconstruire une relation conditionnelle, ou hypothétique, mais à condition que la relation de repérage suive l'ordre linéaire. Ainsi, en (33') ci-dessus, du fait de l'antéposition de la subordonnée en IF, le repérage inter-énoncé suit la même dynamique linéaire qu'avec AND en (33). En revanche l'exemple (33") ci-dessous, avec repère postposé, est pour le moins maladroit, sauf à interpréter la subordonnées comme un ajout a posteriori, après une pause :

(33")??The next thing you'll be telling me is that Stalin should be revered as a great man, if you take your argument one step further.

Dans les exemples suivants (31 et $\left.31^{\prime}\right)$ :

(31) Walk through that room and you found yourself in an altogether different environment. (Auster, Brooklyn, 122)

(31') If you walked through that room, you found yourself in an altogether different environment.

69 On observe également que la substitution du coordonnant AND par le subordonnant IF impose une concordance des repères fictifs de la protase à l'apodose (prétérit en $\mathrm{P}$ et en Q) ce que n'impose pas la structure en AND (détermination minimale au présent en $P$, prétérit dans Q).

Ces observations confirment une différence entre les marqueurs au niveau de l'opération de lien : IF convoque un repère fictif de détermination de $\mathrm{P}$ marqué en tant que cible et base du repérage inter-énoncés. AND, lorsque $\mathrm{P}$ est non asserté, convoque une cible non déterminée situationnellement dans le repérage inter-énoncé, c'est-à-dire que c'est la modalité assertive même de $\mathrm{P}$ qui est ciblée comme repère de celle de $\mathrm{Q}$, et non ses déterminations situationnelles, ce qui autorise des changements de plan entre $\mathrm{P}$ et $\mathrm{Q}$, comme c'est le cas en (31) avec le prétérit sur 'find', et que l'on trouve souvent avec des alternances will/would dans le conjoint $Q$ de ces structures pseudo-impératives en AND.

Les formes schématiques des deux marqueurs demeurent donc bien différenciées.

\section{3. 'Pseudo impératifs' avec OR}

71 Les énoncés interprétés comme 'pseudo-impératifs' coordonnés par OR révèlent également une différence opératoire par rapport à ceux liés par AND, différence attendue, et qui correspond à la forme schématique propre à chacun des coordonnants (voir Fig.1 et Fig. 2 plus haut). Néanmoins, comme pour AND, la construction des valeurs relationnelles $\mathrm{y}$ est aussi issue du niveau de portée de l'opération de coordination. Les paramètres contextuels qui convoquent une cible prédicative ou énonciative pour la coordination avec OR sont nombreux; mais l'interprétation pseudo-conditionnelle montre des récurrences au niveau de la modalité assertive dans $P$ et $Q$, qui centre à chaque fois coordination sur un travail au niveau du calcul des validations des conjoints et de leurs repérages intersubjectifs.

Ainsi, lorsque le premier conjoint construit une évaluation modale de la validabilité d'un événement considéré en un point spécifique du temps (qu'il soit passé, présent ou projeté dans l'avenir), et que le deuxième conjoint reprend anaphoriquement ces modalités de 
validation, alors le coordonnant OR cible le niveau énonciatif, et en particulier la modalité assertive des conjoints, dans la construction du lien.

Considérons quelques exemples:

(34) Mind and have everything ready or there will be a fine fuss. (Ashford, Love, 38.)

(35) "Just call me tonight or I go crazy!" (Lumet, Q\&A)

(35) "You lay off her, you bastard, or you're dead!" (Auster, Brooklyn, 121)

(36) "You keep him out, or else Marina don't work for you no more! She quits! ” (Auster, Brooklyn, 121)

(37) As God is my witness, I'll arrange this glass so that you see cells through it or I'll give up teaching. (Saroyan, Aram, 116.)

(38) He will be running, jumping, shouting, screaming or my name is not Emil Shauffhausen. (Oz, Scoundrels)

(39) They must have liked the apartment, or they wouldn't have stayed so long. (Quirk \& al, CGEL)

Notons que dans ce type d'énoncés, contrairement à ceux coordonnés par AND, le premier conjoint $\mathrm{P}$ est effectivement modalisé, et peut 'tenir tout seul' : qu'il s'agisse de présents simples (35 et 36), d'impératifs (34) ou d'auxiliaires modaux (will en (37) et (38), must en (39), on a affaire à l'assertion d'une visée déontique de $\mathrm{P}$ ou de l'évaluation épistémique de $\mathrm{P}$ par l'énonciateur. Dans tous les cas cependant, les relations prédicatives dans $P$ sont définies seulement comme validables, et non validées. La coordination en OR, conformément à sa forme schématique, opère un retour sur $\mathrm{P}$ pour une ouverture verticale à l'autre valeur possible de sa validation ( $\mathrm{P}^{\prime}$ : you don't have everthing ready; you don't lay off her; you don't keep him out ; they didn't like the appartment etc.), et coordonne cette nouvelle valeur à une représentation autre (there will be a fine fuss, you're dead, they didn't stay long etc.). Il s'agit ici d'une coordination métalinguistique, qui prend pour cible la modalité assertive du premier conjoint. Ainsi, bien que, du fait du type de modalités (déontiques ou épistémiques) inscrites dans $\mathrm{P}$, ce soit la branche positive de la bifurcation notionnelle qui soit envisagée par l'énonciateur, le coordonnant ouvre l'alternative dans un deuxième temps, et c'est la branche négative, associée à un coénonciateur maître de la validation effective, qui est prise comme repère d'une subordination sémantique de P' à $Q$, d'où les gloses en 'otherwise' et les traductions françaises possibles par 'sinon'.

Les interprétations conditionnelles ou corrélatives du coordonnant OR sont ainsi liées à la convocation d'une cible assertive de l'opération d'ouverture à une représentation autre.

Ainsi, les valeurs relationnelles dites 'pseudo conditionnelles' construites dans ces structures coordonnées ne sont pas du tout en contradiction avec les formes schématiques esquissées plus haut pour AND et $\mathrm{OR}$; elles en découlent au contraire très largement. On notera que chaque coordonnant conserve son fonctionnement propre, ce qui amène à des différences très nettes au niveau syntaxique comme sémantique :

- Avec OR, les relations intersubjectives portant sur la relation prédicative dans $\mathrm{P}$ ne sont pas les mêmes qu'avec AND (déontique avec OR / non déontique)

- le point d'anaphore de la coordination n'est pas le même : avec OR, ce qui est repris comme repère d'une subordination sémantique, c'est la branche $\mathrm{P}$ ' des validations possibles, c'est-àdire l'inverse de ce qui est proposé en $\mathrm{P}$, et non la validation de $\mathrm{P}$ comme c'est le cas avec AND.

- la prise en charge modale des énoncés liés, et la relation de dépendance entre les conjoints, sont également très différentes. Avec $\mathrm{OR}$ dans ces structures, $\mathrm{P}$ 'tient tout seul' : l'énonciateur vise la validation de $P$, en dehors de l'énoncé $Q$, et la valuation qualitative de $Q$ est toujours construite comme négative pour le co-énonciateur d'après l'énonciateur, 
contrairement à la structure en AND, où la valuation de $Q$ est laissée à l'appréciation du coénonciateur en relation avec celle de P. L'énonciateur impose avec OR, non seulement le lien de subordination sémantique, mais aussi la valuation qualitative des énoncés liés. ces structures comme cible et repère du calcul de l'assertion et de la validation de $Q$, dans une forme d'assertion complexe où le lien de repérage inter-énoncés lui-même est asserté de façon déontique, comme une injonction de lien nécessaire, ce qui déclenche des interprétations sémantiques particulières des coordonnants, pseudo-conditionnelles ou corrélatives.

Ces coordonnées 'pseudo-impératives' ne sont donc pas des structures à part parce qu'elles construisent une relation de type conditionnel, et n'ont pas de raison d'être traitées comme des exceptions ou des emplois non coordinatifs. Elles mettent en relief de façon intéressante l'interaction des formes dans la sélection d'une cible de l'opération de coordination, et le lien entre la cible sélectionnée et les phénomènes de subordination sémantique construits. Les contraintes linguistiques et récurrences particulières que l'on observe dans ces configurations coordonnées, sont intéressantes au contraire en ce qu'elles révèlent et confirment les formes schématiques différenciées des deux coordonnants, et soulignent l'intérêt d'intégrer, dans ces formes schématiques, l'interface entre les dimensions syntaxique, sémantique et discursive de leur mode opératoire.

\section{Quelques conclusions}

Dans cet article, nous tentons de préciser le cheminement du sens dans les phénomènes de coordination. Dans cette démarche, il semble pertinent de considérer la contribution des paramètres contextuels et situationnels non pas comme des instructions sémantiques directes, mais comme des instructions opérationnelles, dirigeant le niveau de portée des opérations de coordination dans la construction de l'énoncé complexe : 


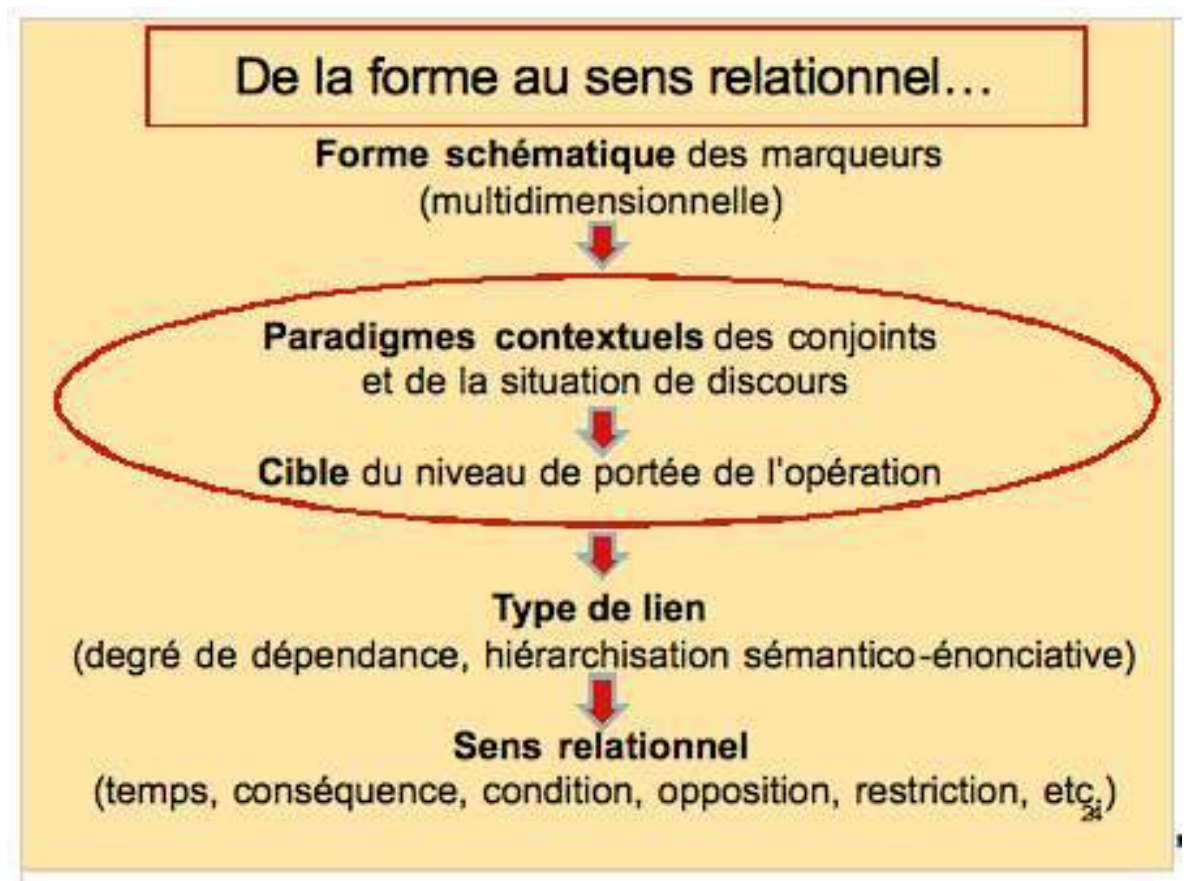

Si chaque coordonnant est la trace d'une opération linguistique propre, d'un 'programme' spécifique de mise en lien des conjoints, dans une relation étroite avec la dynamique linéaire du discours, le coordonnant contient également une dimension intrinsèquement variable. Leur nécessaire interaction avec un ensemble de paramètres, dont quelques paradigmes ont pu être analysés ci-dessus, permet de cibler l'une des strates de la genèse des conjoints à relier, et de tricoter du sens en reprenant, dans la maille, le niveau notionnel prédicatif, le niveau des déterminations et repérages énonciatifs, ou la modalité assertive même des futurs conjoints.

Ainsi, un certain paramétrage du sens relationnel construit par les coordonnants peut être élucidé, et en particulier, la mise en place des relations temporelles ou 'causales', et les interprétations pseudo-impératives des structures coordonnées. Il est certes très ardu, voire illusoire ou contre-productif, de vouloir fixer ces paramètres en une liste exhaustive, du fait de la créativité des combinaisons possibles. Il est intéressant, en revanche, d'observer les schémas d'interactions privilégiées et leur incidence sur la construction et l'interprétation du sens. Les dimensions situationnelles et prosodiques de l'utilisation des coordonnants à l'oral devront d'ailleurs être prises en compte dans une prochaine étude pour compléter cette démarche.

\section{BIBLIOGRAPHIE}

ASHER, N. and VIEU, L. (2005) « Subordinating and coordinating discourse relations". Lingua 115 : 591-610. 
AVANZI, M., LACHERET, A. (à paraître). « Micro-syntaxe, macro-syntaxe : une prosodie toujours transparente ? L'exemple des périodes asyndétique en français parlé ». In : Actes du colloque La Parataxe, M.-J. Béguelin, M. Avanzi \& G. Corminboeuf (eds). Neuchâtel, 2007.

BIERWISCH, M. (2003) Heads, complements, adjuncts: Projection and saturation. In: Modifying adjuncts, E. Lang, C. Maienborn and C. Fabricius-Hansen (eds.), 113-159. Berlin: Mouton de Gruyter.

BLAKEMORE, D. and CARSTON, R. (2005) "The pragmatics of sentential coordination with and." In: R. Carston, and D. Blakemore (eds), LINGUA, Coordination: Syntax, Semantics and pragmatics, pp. 569-590.

BLÜHDORN, H. (2008) Subordination and coordination in syntax, semantics and discourse: Evidence from the study of connectives. In: Fabricius-Hansen, Cathrine \& Ramm, Wiebke (eds.). 'Subordination' versus 'Coordination' in Sentence and Text. Amsterdam, Benjamins, 59-85.

CORMinboeuf, G. (2008) «'Tu m'embrasses encore, et c'est mon pied dans les pompons !' Comment construit-on le sens? » Discours, 3.

COTTE, P. (1992) « Réflexions sur la linéarité », Travaux du CIEREC, 76 :53-76.

CRAKE-ROSSETTE, F. (2003) Parataxe et connecteurs : observations dur l'enchaînement des propositions en anglais contemporain. Thèse de Doctorat.

CRAKE-ROSSETTE, F. (2003) « Le connecteur and : explicitation ou parataxe? », Anglophonia Sigma $14: 79-100$.

CRISTOFARO, S. (2003) Subordination. Oxford: University Press.

CULICOVER, P., JACKENDOFF, R. (1997) “Semantic subordination despite syntactic coordination.” Linguistic Inquiry 28:195-217.

CULICOVER, P., JACKENDOFF, R. (2005) Simpler syntax. Oxford-New-York: Oxford University Press. CULIOLI, A., (2002) Pour une linguistique de l'énonciation, Tomes I\&II, Paris, Ophrys.

DANCYGIER, B., SWEETSER, E. (2006) Mental Spaces in Grammar : conditional constructions. Cambridge : CUP.

DELECHELLE, G. (1994) «Relations inter-énoncés : de la subordination à la coordination », RANAM XXVII : 29-41.

DE VOGÜÉ, S. (1999) «Le champ des subordonnées dites conditionnelles du français : conditions, éventualités, suppositions et hypothèses ", LINX, 41 : 93-118.

DE VOGÜÉ, S. (2000) «Calcul des valeurs d'un énoncé `au présent'« , Travaux de linguistique, 40 : 31-54.

FRANKE, M. (2008) "Pseudo imperatives and other cases of conditional conjunctions and conjunctive disjunctions”. In: Subordination versus coordination, Sentence and Text. Fabricius-Hensen \& Ramm Edts, Studies in Language Companion Series 98, John Benjamins Publishing Company.

GOURNAY, L. (1998) Étude contrastive de la coordination en français et en anglais, Ophrys, Gap.

GOURNAY, L. (2006) « Peut-on parler d'emplois non-coordinatifs des coordonnants? », Article en ligne sur le site de l'ALAES, document PDF.

GOURNAY, L. (2007) « Les formes de la coordination notionnelle en français et en anglais ». In : M-L. Groussier \& C. Rivière. Cahier de Recherche de Grammaire Anglaise, tome 10. Paris: Ophrys 
LANG, E. (1984) The semantics of coordination. Engl. transl. by John Pheby. Amsterdam: John Benjamins.

LANGACKER, R.W. 1991. Foundations of Cognitive Grammar. vol. 2: Descriptive application. Stanford : Stanford University Press.

LARCHER, P. (1992) « De Bally à Ducrot : note sur les concepts de 'coordination' et 'subordination' sémantiques ", La subordination, Travaux du CERLICO, 5 : 29-42.

PERRIN, I. (1992) “'Think of Baked Beans and You Think of Heinz': les impératifs dits conditionnels”. In : I. Perrin (ed), Approches énonciatives de l'énoncé complexe, L'Information grammaticale, Louvain, Paris : Éditions Peeters.

POLANYI, L. (1988) “A formal model of the structure of discourse”, Journal of Pragmatics 12:601-638. QUIRK R., GREENBAUM, S., LEECH, G. AND SVARTVIK, J. (1985) A Comprehensive Grammar of the English Language, Longman, chapter 13, pp. 918-988.

ROCQ-MIGETTE, C. (2005) « La construction de l'interdépendance : structures conditionnelles du type , p or/ou q $>$ ». Colloque Subordination - Coordination. Accessible en ligne sur : http:// www.cavi.univ-paris3.fr/ilpga/colloque-coord-subord-2005/pre-textes/.

SEKALI, M. (1991) «Connexion inter-énoncés et relations intersubjectives : because, since et for », LANGAGES, $104: 62-78$.

SEKALI, M. (1992) « Subordination temporelle et subordination subjective : l'exemple de since », Travaux du CERLICO, Subordination/Subordinations, 5 :105-126

SEKALI, M. (2007) “'He's a cop but he isn't a bastard': an enunciative approach to some pragmatic effects of the coordinator but". In: A. Celle \& R. Huart (eds), Connectives as Discourse Landmarks, John Benjamins Publishing Company, Pragmatics \& Beyond New Series, 161, pp. 155-175.

SEKALI, M. (2010) Coordination et dynamique discursive : étude comparative des coordonnants anglais and, or, but et for, in DIRECTIONS ACTUELLES EN LINGUISTIQUE DU TEXTE, Florea, Papahagi, Pop, \& Curea Eds, Casa CărŢii de ştiinŢă, Cluj-Napoca, pp. 235-245

SEKALI, M. (sous presse) Counter-subordinative coordination vs. co-subordinative coordination: a syntax/semantics interface study of AND, BUT and FOR, in INTERFACES IN LANGUAGE 2, Newcastle upon Tyne: Cambridge Scholars Publishing.

TRÉVISE, A. (ed). (1999). L'hypothétique, LINX 41.

TRÉVISE, A. \& CONSTANT, L. (2007). « Asyndète et construction du fictif dans certaines variétés d'anglais ». In : A. Celle, S. Gresset \& R. Huart (eds), Les connecteurs, jalons du discours, pp. 71-94.

\section{Oeuvres et corpora cités :}

Browns Corpus (BROWNS)

British National Corpus (BNC)

Corpus of Contemporary American English (COCA)

Ashford, D. Love and Marriage, R. Hartt Davis Ltd. London, 1965.

Auster, P., The Brooklyn Follies, Picador USA; Reprint 2006.

Saroyan, W., My Name is Aram, Penguin Books, USA, 1945. 
Wharton, E., The Custom of the Country, 1913; The Echo Library USA; 2005.

Films:

Levinson, B, Rain Man, 1989

Kubrick, S. The Killing, 1956.

Lumet, S. Questions \& Answers, 1990.

Oz, F., Dirty Rotten Scoundrels, 1988)

\section{RÉSUMÉS}

Les coordonnants AND et $\mathrm{OR}$ sont à la fois polyvalents (pouvant construire des relations conceptuellement hiérarchisées ou non hiérarchisées) et polysémiques (marquant la surenchère, la consécution, la conséquence, la condition etc.). Cet article tente de formaliser le paramétrage du sens des deux coordonnants en partant de cette double variabilité intrinsèque. Des familles de paramètres contextuels et situationnels sont relevées dans leur interaction privilégiée avec la forme schématique de chaque coordonnant, et en tant qu'ils sélectionnent, non pas directement la valeur sémantique de la relation, mais la cible (ou portée) de l'opération de coordination. Sont analysées ici les instructions de ciblage des niveaux prédicatif, énonciatif, ou assertif de la structuration des conjoints dans la mise en lien, et leur incidence sur la genèse du sens relationnel, dans les structures coordonnées interprétées comme sémantiquement symétriques ou au contraire hiérarchisées et dans les coordonnées pseudo-impératives.

English coordinators AND and OR can be considered as cases of linguistic polyvalence (constructing symmetrical or a-symmetrical relations) and polysemy (mapping a number of semantic notions such as escalation, temporal sequence, consequence, condition etc.). This paper aims at formalizing patterns of meaning construction for the two coordinators, starting from their intrinsic double variability. Paradigms of contextual and situational variables are observed in the way they interact with the coordinators - not to directly map their relational meaning but to instruct and specify the target of the coordination process. Such targeting instructions are analyzed in a variety of coordinated structures which are interpreted as conceptually symmetrical, non-symmetrical or pseudo-imperative, according to the selection, as the scope of the combining process, of either the level of bare predication within the conjuncts, or their situational determinations or even their assertive modalities.

\section{INDEX}

Mots-clés : coordination, polyvalence, instructions de cible, construction du sens

Keywords : targeting instructions, semantic mapping

\section{AUTEUR}

\section{MARTINE SEKALI}

Université Paris-Ouest, Nanterre, CREA-GREG

sekali@u-paris10.fr 